\title{
Evidence for TeV gamma ray emission from TeV J2032+4130 in Whipple archival data
}

\author{
M. J. Lang ${ }^{1}$, D. A. Carter-Lewis ${ }^{2}$, D. J. Fegan ${ }^{3}$, S. J. Fegan ${ }^{4}$, A. M. Hillas ${ }^{5}$, R. C. Lamb ${ }^{6}$, M. Punch ${ }^{7}$, \\ P. T. Reynolds ${ }^{8}$, and T. C. Weekes ${ }^{4}$ \\ 1 Department of Physics, National University of Ireland, Galway, Ireland \\ e-mail: mark. lang@nuigalway.ie \\ 2 Department of Physics and Astronomy, Iowa State University, Ames, IA 50011, USA \\ 3 Department of Experimental Physics, University College, Belfield, Dublin 4, Ireland \\ ${ }^{4}$ Fred Lawrence Whipple Observatory, Harvard-Smithsonian CfA, Amado, AZ 85645, USA \\ 5 Department of Physics and Astronomy, University of Leeds, Leeds, LS2 9JT, UK \\ ${ }^{6}$ Space Radiation Laboratory, California Institute of Technology, Pasadena, CA 91125, USA \\ 7 Physique Corpusculaire et Cosmologie, Collège de France, 11 place Marcelin Berthelot, 75231 Paris Cedex 05, France \\ 8 Department of Applied Physics and Instrumentation, Cork Institute of Technology, Bishopstown, Cork, Ireland
}

Received 5 April 2004 / Accepted 12 May 2004

\begin{abstract}
A reanalysis of data taken on the Cygnus region in 1989-90 using the Whipple Observatory atmospheric Cherenkov imaging telescope confirms the existence of the TeV J2032+4130 source reported by the Crimean Astrophysical Observatory and published by the HEGRA Collaboration. The significance at the a priori HEGRA position is $3.3 \sigma$. The peak signal was found at RA $=20 \mathrm{~h} 32 \mathrm{~m}$, Dec $=+41^{\circ} 33^{\prime}$. This is $0.6^{\circ}$ north of Cygnus X-3 which was the original target of the observations. The flux level (12\% of the level of the Crab Nebula) is intermediate between the two later observations and suggests that the $\mathrm{TeV}$ source is variable.
\end{abstract}

Key words. gamma rays: observations

\section{Introduction}

The most powerful technique for the discovery of cosmic sources of $\mathrm{TeV}$ gamma rays is the atmospheric Cherenkov technique using cameras consisting of arrays of photomultiplier tubes coupled to large optical reflectors. Such instruments have fields of view that are typically less than $5^{\circ}$ and hence are not suitable for sky surveys. The usual mode of operation is to make directed observations of candidate objects (chosen from their properties at longer wavelengths) and more than a dozen sources have been thus established (Horan \& Weekes 2004). Sky surveys at these energies are comparatively insensitive and have not resulted in the detection of any new sources (Weekes et al. 1979; Atkins et al. 2004; Cui et al. 2003). By contrast the $100 \mathrm{MeV}$ space telescopes are ideally suited for sky surveys and have discovered some 270 discrete sources; more than half the sources detected by the EGRET telescope on the Compton Gamma Ray Observatory are unidentified (Hartman et al. 1999)

The increasing sensitivity of the ground-based gammaray telescopes and the ability to make two-dimensional maps of the field of view encompassed by the camera makes possible serendipitous detection of $\mathrm{TeV}$ gamma-ray sources in the vicinity of candidate objects or in the control regions commonly used in making such observations. Regions of the sky that include the Galactic Plane are particularly attractive for such searches. Early indications that Cygnus X-3 might be a $\mathrm{TeV}$ source (Vladimirsky et al. 1973), led to a concentration of observations in that crowded region of the sky. Recent observations have not confirmed emission from Cygnus X-3 but a two-dimensional study by the Crimean group of observations taken in 1993 suggested the existence of new source offset by $0.7^{\circ}$ from Cygnus X-3 (Neshpor et al. 1995). The signal was at the $6 \sigma$ level before taking trial factors into account. The observations were made over a six week period and correspond to a flux of $3 \times 10^{-11}$ photons $\mathrm{cm}^{-2} \mathrm{~s}^{-1}$ above an energy of $1 \mathrm{TeV}$; this is a strong source at approximately the level of the Crab Nebula, the strongest known steady source in the sky. The coordinates of the new source were Right Ascension = $20 \mathrm{~h} 32 \mathrm{~m}$ and Declination $=+41^{\circ} 37^{\prime}$. The uncertainty in the source position was estimated as $0.2^{\circ}$. Subsequent observations by the Crimean group in the following years did not confirm the emission at this high level (Fomin 2004).

In a series of observations by the HEGRA group (Aharonian et al. 2002), the source was independently and serendipitously detected in observations of Cygnus X-3 and the EGRET unidentified source, GeV J2035+4214 
(Lamb \& Macomb 1997). Both sets of observations encompassed the position of the Crimean source. A detection was reported based on $113 \mathrm{~h}$ of observation over a three year observing campaign (1999-2001 inclusive) at the $4.6 \sigma$ post-trial level of significance. This large database was expanded to $279 \mathrm{~h}$ with additional observations taken in 2002 and the statistical significance was increased to the $7 \sigma$ level (Rowell et al. 2003). The source location was determined to be Right Ascension $(\mathrm{J} 2000)=20 \mathrm{~h} 31 \mathrm{~m} 57.0 \mathrm{~s}$, Declination $(\mathrm{J} 2000)=+41^{\circ} 29^{\prime} 56.8^{\prime \prime}$ with statistical and systematic uncertainties $\approx$ a few minutes of arc. This position is consistent with that of the Crimean source. However the flux level was only about $3 \%$ of the Crab flux $\left(F(>1 \mathrm{TeV})=(5.9 \pm 3.1) \times 10^{-13}\right.$ photons $\left.\mathrm{cm}^{-2} \mathrm{~s}^{-1}\right)$ and the differential spectral index was hard $(-1.9)$. The source was reported to be extended $\left(6.2^{\prime}\right)$ with a point source hypothesis rejected at the $3 \sigma$ level. However, this is a complicated region of the Galactic Plane with three EGRET sources nearby but not obviously related. The source lies close to the edge of the dense OB association, Cygnus OB2.

Following these reports we were motivated to reanalyze data taken on Cygnus X-3 with the Whipple telescope at an earlier epoch and at a somewhat lower energy threshold to search for evidence of this source.

\section{Observations}

Cygnus X-3 was observed using the Whipple Observatory $10 \mathrm{~m}$ imaging telescope during the epoch 1988-1990, (O'Flaherty et al. 1992). The observations were carried out in the ON/OFF tracking mode, with Cygnus X-3 located at the centre of the field of view. In this mode the telescope tracks the object for $38 \mathrm{~min}$, recording an ON scan. Then during a 2 min interval the telescope is slewed to a position 40 min later in Right Ascension and records a comparison OFF scan over the same range of zenith and azimuth angles. The telescope was equipped with a 109-pixel camera, consisting of 91 phototubes of diameter $2.9 \mathrm{~cm}$ (with a pixel separation of $0.25^{\circ}$ ), surrounded by an outer ring of 18 phototubes with a diameter of $5 \mathrm{~cm}$ (with a pixel separation of $0.5^{\circ}$ ), (Cawley et al. 1990). The field of view of the camera was $3.5^{\circ}$ which includes the reported location of $\mathrm{TeV} \mathrm{J} 2032+4130$. The signal in each phototube was amplified and converted to a digital value in which each digital count represented a signal of 1.15 photoelectrons. The camera was triggered when the signal in 2 out of the inner 91 phototubes exceeded a threshold of about 40 digital counts. Moment-fitting parameters were used to characterise the shape and orientation of each recorded Cherenkov image. Gamma-ray-like events were selected on the basis of their shape and orientation defined in a single parameter, azimuthal width (azwidth) (Vacanti et al. 1991). During the season 1988-1989 this instrument was used to detect steady $\mathrm{TeV}$ emission from the Crab Nebula at the $20 \sigma$ level. No evidence was found by O'Flaherty et al. (1992) for steady, modulated, or pulsed emission from Cygnus X-3. A two-dimensional analysis of the field of view was not carried out at the time.

After each night's observations, the data were archived as binary files on magnetic tapes for subsequent analysis. During the early 1990's the entire 109-pixel camera database was
Table 1. Summary of data.

\begin{tabular}{cccccc}
\hline \hline Year & No. of data pairs & $\begin{array}{c}\text { Duration } \\
(\mathrm{h})\end{array}$ & $N($ on $)$ & $N($ off $)$ & $\begin{array}{c}S \\
(\sigma)\end{array}$ \\
\hline 1989 & 43 & 26.8 & 367783 & 368508 & -0.8 \\
1990 & 40 & 23.5 & 280759 & 280904 & -0.2 \\
Total & 83 & 50.4 & 648542 & 649412 & -0.8 \\
\hline
\end{tabular}

Table 2. Supercuts selection criteria.

\begin{tabular}{ll}
\hline \hline Trigger & 2 phototubes $>40$ digital counts \\
Shape & $0.51^{\circ}<$ Distance $<1.1^{\circ}$ \\
& $0.073^{\circ}<$ Width $<0.15^{\circ}$ \\
& $0.16^{\circ}<$ Length $<0.30^{\circ}$ \\
Orientation & $0.51^{\circ}<$ Distance $<1.1^{\circ}$ \\
& Alpha $<15^{\circ}$ \\
\hline
\end{tabular}

transferred to digital tape (DAT). These DAT's formed the data archive for the subsequent analysis. Table 1 summarises the database used in this analysis. Note that the precise list of the data files originally used by O'Flaherty et al. (1992) in the search for emission from Cygnus X-3 was no longer available.

In the reanalysis a small amount of the original data recorded in 1988 was omitted due to the elevated levels of electronic noise present. Slightly more data for the years 1989 and 1990 was included in the analysis. This initial selection of data was made prior to the search for gamma-ray sources. The average elevation of the observations was about $70^{\circ}$. The statistical significance $(S)$ of any excess of events seen in the ON region is calculated using the combined uncertainty in the ON and OFF event numbers ( $\mathrm{Li} \& \mathrm{Ma} 1983$ ). There is no evidence for an excess of events from Cygnus X-3 in the unselected (raw) data.

\section{Analysis}

As the Cherenkov imaging technique evolved, a more effective set of gamma-ray image criteria known as supercuts was developed, which separately utilised the shape and orientation information in the image. The Crab database of 198889 was used to optimise the parameters in supercuts (Punch et al. 1991) and the criteria were subsequently applied to observations of the Crab Nebula from the 1989-90 and 1990-91 seasons giving significant improvements in sensitivity (Lang et al. 1991). Supercuts was then adopted as the standard analysis technique for observations with this camera (Reynolds et al.1993), and, with minor modifications, has remained the standard selection method for subsequent higher resolution instruments. The supercuts selection criteria are listed in Table 2. A standard supercuts analysis of the database was made in which the image distance and alpha parameters were calculated relative to the source position reported by Rowell et al. (2003). It was assumed that the optic axis of the camera was centred on Cygnus X-3. After de-rotation of the field of view by the parallactic angle, this location is displaced from the 
Table 3. Results for HEGRA location.

\begin{tabular}{lcccc}
\hline \hline & $N($ on $)$ & $N($ off $)$ & $N($ on $)-N($ off $)$ & $S$ \\
\hline Shape & 12333 & 12446 & -113 & -0.72 \\
Orientation & 42607 & 42692 & -85 & -0.29 \\
Shape+Orientation & 2031 & 1824 & +207 & +3.33 \\
\hline
\end{tabular}

centre of the field of view by $0.09^{\circ}$ in the $x$-axis (corresponding to Right Ascension) and by $0.54^{\circ}$ in the $y$-axis (corresponding to Declination). The results are listed in Table 3. There is a $3.3 \sigma$ excess present at this location. As this is an a priori search location there are no trial factors to be included and the presence of a weak $\mathrm{TeV}$ source is confirmed.

A two-dimensional search technique has previously been applied to the Crab Nebula database of 1988-89 by Akerlof et al. (1991). The field of view was divided using a Cartesian grid system covering an interval of $\pm 1.0^{\circ}$ in both the Right Ascension and Declination directions. The grid step size was $0.1^{\circ}$, giving a total of 441 mesh points. An azwidth-type analysis was carried out at each mesh point and the Crab Nebula signal appeared as an approximately two dimensional Gaussian function with a circular probable error of radius $0.21^{\circ}$. This is the gamma-ray point spread function (PSF) of the instrument. A similar analysis was carried out for observations in which the Crab Nebula was deliberately offset by $0.4^{\circ}$ and $1.0^{\circ}$ from the centre of the field of view. In each case a signal was detected. The efficiency of the telescope (measured in $\sigma \mathrm{hr}^{-1}$ ) was undiminished when the source was offset by $0.4^{\circ}$ and was reduced to $45 \%$ for the offset of $1.0^{\circ}$. At each offset the source location reconstructed from the shower images agreed with the tracking location to within approximately $0.03^{\circ}$, with the dominant error attributed to the telescope angle encoders and drive system.

We applied this two-dimensional analysis to the Cygnus X-3 database, where this time events were selected using the supercuts criteria at the 441 mesh points. Figure 1 is a sky map of the ON minus OFF signal over the grid. The significance of the pre-trial peak signal is $4.0 \sigma$, corresponding to an excess of 242 events. The peak occurs at the grid point $0.0^{\circ}$ in the Right Ascension direction and $+0.6^{\circ}$ in Declination, $\left(\mathrm{RA}_{J 2000}=20 \mathrm{~h} 32 \mathrm{~m}, \operatorname{Dec}_{J 2000}=+41^{\circ} 33^{\prime}\right)$. which is compatible with both the Crimean and HEGRA positions. As a conservative estimate of the number of trial factors introduced by searching "near" the HEGRA position we have used the value 7, which corresponds to a search over one quarter of the field of view. This reduces the significance of the peak to $3.5 \sigma$. If a linear fall-off in sensitivity with displacement over the interval of $0.4^{\circ}$ to $0.6^{\circ}$ from the centre of the field of view is assumed, the gamma-ray rate corresponds to $12 \%$ of the Crab flux, $\left(5.6 \pm 1.6_{\text {stat }} \pm 0.8_{\text {sys }}\right) \times 10^{-12}$ photons $\mathrm{cm}^{-2} \mathrm{~s}^{-1}$, above the peak energy response of $0.6 \mathrm{TeV}$. The systematic error is representative of the uncertainty in the off-centre sensitivity of the telescope.

There is no evidence that the signal is variable over the two years of observation. Given the gamma-ray PSF value and the weak nature of the signal, we are unable to determine the angular size of the source.

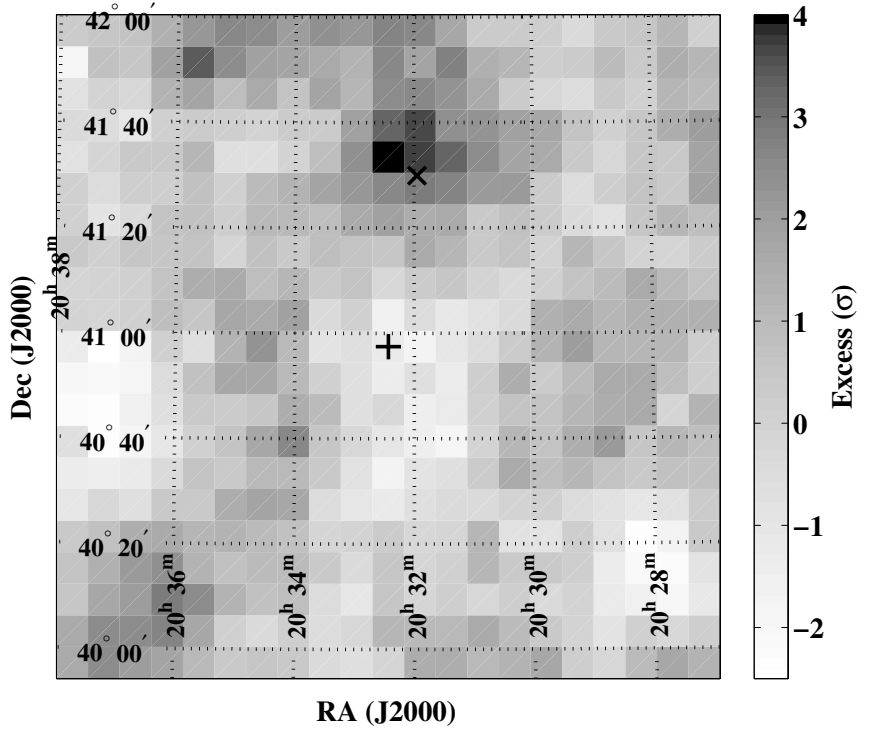

Fig. 1. Sky map of the excess significance $(\sigma)$ in a $2^{\circ} \times 2^{\circ}$ region centred on Cygnus X-3 (marked with $\mathrm{a}+$ ). The HEGRA position for $\mathrm{TeV} \mathbf{J} 2032+4130$ is marked with an $\times$.

Table 4. Peak signal results selected by image size (pre-trials).

\begin{tabular}{ccccc}
\hline \hline & $N($ on $)$ & $N($ off $)$ & $N($ on $)-N($ off $)$ & $S$ \\
\hline \hline Size $\leq 600 \mathrm{dc}$ & 1574 & 1405 & 169 & +3.10 \\
Size $>600 \mathrm{dc}$ & 395 & 322 & 73 & +2.73 \\
\hline
\end{tabular}

Although the signal is too weak to allow the determination of an energy spectrum we have investigated the effect of raising the energy threshold in software. The image parameter size measures the total number of photoelectrons in a Cherenkov image, and therefore the gamma-ray energy. Table 4 lists the results when the data are selected on the basis of image size. The cutoff point of 600 digital counts corresponds to a doubling of the peak energy response to about $1.2 \mathrm{TeV}$. It would appear that the signal is not confined to low energy events.

The use of software padding procedures, in which Gaussian noise is added in order to equalise the night-sky noise in the ON and OFF regions, was not part of the standard analysis technique for the 109-pixel camera when this data was taken. Padding was only used in cases of "extreme" brightness differences (Punch et al. 1991). The Cygnus X-3 ON and OFF regions have similar sky brightness. The night-sky noise in each phototube typically had a standard deviation of $3 \sim 4$ photoelectrons, with the difference between the ON and OFF regions having an average value of 0.05 photoelectrons. However, given the weakness of the signal detected from $\mathrm{TeV} \mathrm{J} 2032+4130$, the effect of software padding on the database was investigated. Where $\sigma_{\mathrm{ON}}$ and $\sigma_{\mathrm{OFF}}$ are the standard deviations of the sky noise in a particular phototube in the $\mathrm{ON}$ and $\mathrm{OFF}$ regions respectively, the standard deviation of noise to be added in the less noisy region is given by

$\sigma_{\mathrm{add}}=\sqrt{\left|\sigma_{\mathrm{ON}}^{2}-\sigma_{\mathrm{OFF}}^{2}\right|}$.

Software padding is discussed in detail by Lessard et al. (2001). The two-dimensional analysis was repeated ten times, where in 


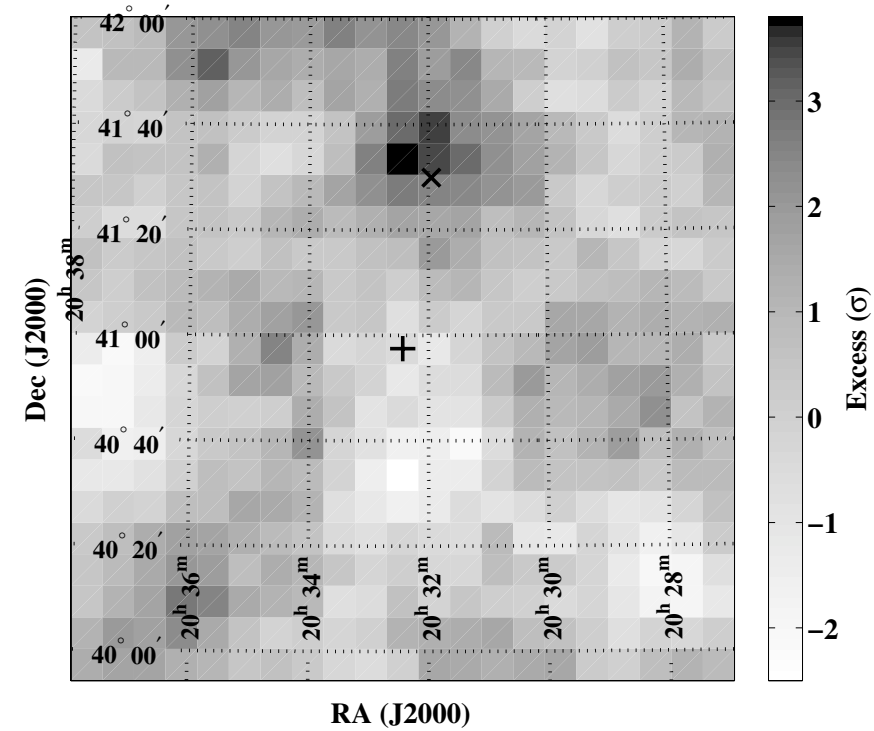

Fig. 2. Sky map of the excess significance $(\sigma)$ in a $2^{\circ} \times 2^{\circ}$ region centred on Cygnus X-3 (marked with a + ), after the application of software padding. The HEGRA position for TeV J2032+4130 is marked with an $x$.

each case software padding using a different seed for the random number generator was applied. In order to more closely reproduce the effect of real night-sky noise on the event trigger rate, the trigger threshold was raised to two phototubes exceeding 45 digital counts. This creates a population of sub-threshold events which may trigger the system after the addition of noise. In each case a peak was found at the HEGRA position. Because a random feature was introduced into the analysis, the pre-trials peak significance varies between $3.1 \sigma$ and $4.5 \sigma$. To produce a robust padded analysis the average number of events from the ten analyzes which pass the supercuts criteria at each mesh point was calculated. These averages were then used to determine the ON minus OFF significance at each mesh point. The resulting sky map is illustrated in Fig. 2, and is very similar to the sky map for the unpadded data, confirming that the effect seen does not result from a difference is night-sky brightness. The significance of the pre-trials peak is $3.8 \sigma$. Given that some noise has been added and the trigger threshold has been increased, this slight reduction in significance is not unexpected.

A subset of the database, comprising 24 out of 83 data pairs, was recorded in the OFF-before-ON mode, where the OFF region was $40 \mathrm{~min}$ ahead in Right Ascension. The average ON minus OFF pre-trials signal per pair in the OFF-before-ON data is $(0.39 \pm 0.20) \sigma$ compared with $(0.46 \pm 0.13) \sigma$ in the ON-before-OFF data. Again this indicates that the signal is not a sky-noise artifact.

\section{Discussion}

TeV J2032+4130 is located within the crowded Cygnus region of the Galactic Plane and hence it has no shortage of possible candidate identifications. The region is dominated by the very strong OB association, Cygnus OB2 and this was the first identification suggested (Aharonian et al. 2002). At a distance of $1.7 \mathrm{kpc}$, this implies a luminosity in the range $10^{32-34} \mathrm{erg}$ which is a small fraction of the total energy estimated for the $\mathrm{OB}$ association. The apparent finite size of the $\mathrm{TeV}$ source would favour this identification.

An extensive study of the source region by Butt et al. (2003) using the Chandra and VLA telescopes was made to examine the hypothesis that the large scale shocks and turbulence induced by multiple interacting stellar winds from the young stars in the OB association might accelerate the hadronic component of the cosmic radiation and cause the emission of gamma rays. It was assumed that the $\mathrm{TeV}$ source was extended and steady. This work has been extended by Torres et al. (2004) who predict that this may be a strong source of $\mathrm{TeV}$ neutrinos.

An alternative approach was taken by Mukherjee et al. (2003) who associated $\mathrm{TeV} \mathrm{J} 2032+4130$ with the nearby EGRET unidentified source 3EG J2033+4118 and assumed the $\mathrm{TeV}$ source to be variable and point-like. They suggested an identification with the brightest X-ray source in the field which is variable and has a featureless spectrum suggestive of a BL Lac. This was suggested to be a "proton blazar" which would be notable for having little radio emission.

Other possible identifications include an unusual supernova remnant caused by a supernova some tens of thousands of years ago (Bednarek 2004) and the jet from the nearby microquasar, Cygnus X-3 (Aharonian et al. 2002).

The detection of a relatively weak signal at a somewhat lower peak energy and at an earlier epoch is significant in that it verifies that the source is variable and hence aids in its identification with candidate objects. The detection is not deep enough to improve the positional information or to better define the energy spectrum. Also it is not possible to determine the angular size of the source which, like the other $\mathrm{TeV}$ sources detected by these experiments, is consistent with a point source. However the average flux detected in 1989-1990 (12\% of the Crab) is clearly well above that seen as the average flux over a four year period by the HEGRA group (3\% of the Crab) in 1999-2002 and well below that seen over a six week period in 1993 by the Crimean experiment (approximately the level of the Crab). The low level of emission from $\mathrm{TeV} \mathrm{J2032+4130} \mathrm{in} \mathrm{the} \mathrm{current} \mathrm{epoch} \mathrm{is} \mathrm{confirmed} \mathrm{by} \mathrm{ob-}$ servations at the Whipple Observatory in 2003 (Finley 2003). Neither the Whipple nor the HEGRA experiments see any evidence for variability within their individual databases. The large differences between the flux levels cannot be explained as errors in estimation of the sensitivity of the three experiments since they have been calibrated by the simultaneous observations of other $\mathrm{TeV}$ sources.

The observed variability is difficult to reconcile with the HEGRA observation that the source is extended. It is unfortunate that the two early experiments did not have sufficient depth in their detections to confirm this extension. The variability seen is easier to explain in terms of a point source such as the proton blazer or the microquasar explanations. More sensitive observations by VERITAS and GLAST over the full energy range will probably be needed to unambiguously unravel the nature of the source. The correlation of time variations with observations at longer wavelengths will be particularly important. 
Acknowledgements. The contributions of M. F. Cawley, K. S. O'Flaherty and G. Vacanti to the observational programme, and to the development of the original analysis software, are gratefully acknowledged.

\section{References}

Aharonian, F., Akhperjanian, A., Beilicke, M., et al. 2002, A\&A, 393, L37

Akerlof, C. W., Cawley, M. F., Chantell, M., et al. 1991, ApJ, 377, L97

Atkins, R., Benbow, W., Berley, D., et al. 2004, ApJ, 608, 680

Bednarek, W. 2003, MNRAS, 345, 847

Butt, Y. M., Benaglia, P., Combi, J. A., et al. 2003, ApJ, 597, 494

Cawley, M. F., Fegan, D. J., Harris, K., et al. 1990, Exp. Astron., 1, 173

Cui, S. W., \& Yan, C. T. (on behalf of the Tibet AS Gamma Collaboration) 2003, Proc. 28th ICRC (Tsukuba), 4, 2315

Finley, J. P. 2003, BAAS, A\&AS 203rd Meet. (Atlanta, 2004), 35, 1216

Fomin, V. 2004, private communication

Hartman, R. C., Bertsch, D. L., Bloom, S. D., et al. 1999, ApJS, 123, 79

Horan, D., \& Weekes, T. C. 2004, New Astron. Rev., 48, 527
Lamb, R. C., \& Macomb, D. 1997, ApJ, 488, 872

Lang, M. J., et al. 1991, Proc. 22nd ICRC (Dublin), 1, 204

Lessard, R. W., Buckley, J. H., Connaughton, V., \& Le Bohec, S. 2001, Astropart. Phys., 15, 1

Li, T. P., \& Ma, Y. Q. 1983, ApJ, 272, 317

Mukherjee, R., Halpern, J. P., Gotthelf, E. V., Eracleous, M., \& Mirabal, N. 2003, ApJ, 589, 487

Neshpor, Yu. I., Kalekin, O. R., Stepanian, A. A., et al. 1995, Proc. 25th ICRC (Rome), 2, 385

O’Flaherty, K. S., Cawley, M. F., Fegan, D. J., et al. 1992, ApJ, 396, 674

Punch, M., Akerlof, C. W., Cawley, M. F., et al. 1991, Proc. 22nd ICRC (Dublin), 1, 464

Reynolds, P. T., Akerlof, C. W., Cawley, M. F., et al. 1993, ApJ, 404, 206

Rowell, G., \& Horns, D. (for the HEGRA Collaboration) 2003, Proc. 28th ICRC (Tsukuba), 4, 2345

Torres, D. F., Domingo-Santamaria, E., \& Romero, G. E. 2004, ApJ, 601, L75

Vacanti, G., Cawley, M. F., Colombo, E., et al. 1991, ApJ, 377, 467

Vladimirsky, B. M., Stepanian, A. A., \& Fomin, V. P. 1973, Proc. 13th ICRC (Denver), 1, 456

Weekes, T. C., Helmken, H., \& Horine, E. 1979, Proc 16th ICRC (Kyota) 1, 126 